\title{
Re: Mesh, Graft, or Standard Repair for Women Having Primary Transvaginal Anterior or Posterior Compartment Prolapse Surgery: Two Parallel-Group, Multicentre, Randomised, Controlled Trials (PROSPECT)
}

Glazener $\mathrm{CM}^{1}$, Breeman $\mathrm{S}^{1}$, Elders $\mathrm{A}^{2}$, Hemming $\mathrm{C}^{3}$, Cooper $\mathrm{KG}^{3}$, Freeman $\mathrm{RM}^{4}$, Smith $\mathrm{AR}^{5}$, Reid $\mathrm{F}^{5}$, Hagen $\mathrm{S}^{2}$, Montgomery $\mathrm{I}^{1}$, Kilonzo $\mathrm{M}^{6}$, Boyers $\mathrm{D}^{7}$, McDonald $\mathrm{A}^{1}$, McPherson $\mathrm{G}^{1}$, MacLennan $\mathrm{G}^{1}$, Norrie $\mathrm{J}^{1}$, (PROSPECT study group)

${ }^{1}$ University of Aberdeen, Health Services Research Unit, Aberdeen, UK

${ }^{2}$ Glasgow Caledonian University, Nursing, Midwifery and Allied Health Professions Research Unit, Glasgow, UK

${ }^{3}$ Aberdeen Royal Infirmary, Clinic of Obstetrics and Gynaecology, Aberdeen, UK

${ }^{4}$ Plymouth Hospitals NHS Trust, Clinic of Obstetrics and Gynaecology, Plymouth, UK

${ }^{5}$ Central Manchester University Hospitals NHS Foundation Trust, Manchester Academic Health Science Centre, St Mary's Hospital, Warrell Unit,

Manchester, UK

${ }^{6}$ University of Aberdeen, Health Economics Research Unit, Aberdeen, UK

${ }^{7}$ University of Aberdeen, Health Services Research Unit, Health Economics Research Unit, Aberdeen, UK

Lancet 2017;389:381-392. doi: 10.1016/S0140-6736(16)31596-3.

\section{EDITORIAL COMMENT}

This study was designed to compare the outcomes of prolapse repair surgery in randomly assigned groups of non-absorbable synthetic mesh and biological grafts against standard repair with native tissue. The primary focus was patient-reported outcomes and their experience of adverse effects. Two groups were formed for comparison; 865 women were included in the mesh trial group (430 assigned to standard repair alone, 435 to mesh augmentation) and 735 subjects were included in the graft trial group (367 assigned to standard repair alone, 368 to graft augmentation). The authors measured outcomes by participant-completed postal questionnaire at baseline (before surgery), at 6 months, 1 year, and 2 years after surgery and in a clinic review appointment at 1 year (with the Pelvic Organ Prolapse Quantification system). The primary clinical outcome which was defined as woman's report of prolapse symptoms was assessed using the Pelvic Organ Prolapse Symptom Score (POP-SS).

There was no difference in mean POP-SS at 6 months, 1 year and 2 years, and in mean European Quality of Life-5 Dimensions 3-level scores between the groups. Overall, less than 10\% of women had serious complications after prolapse surgery in the first year with no significant difference between the trial groups except for mesh exposure, but interestingly, the extrusion of mesh into the vagina was small or asymptomatic and there was no difference in dyspareunia rates with or without mesh or biological graft.

The authors concluded that this study showed that augmenting a primary transvaginal prolapse repair with synthetic mesh or biological graft offers no benefit over standard repairs. They also considered the risk for additional surgical procedure need for mesh exposures or extrusions in the first 2 years and suggested that mesh usage could be limited to predefined special groups like high-risk women.

Metin Onaran, MD

๑Copyright 2017 by the Association of Urological Surgery / Journal of Urological Surgery published by Galenos Publishing House. 